\title{
Covering Vectors by Spaces: Regular Matroids*
}

\author{
Fedor V. Fomin ${ }^{1}$, Petr A. Golovach ${ }^{2}$, Daniel Lokshtanov ${ }^{3}$, and \\ Saket Saurabh ${ }^{4}$
}

1 Department of Informatics, University of Bergen, Bergen, Norway

fedor.fomin@ii.uib.no

2 Department of Informatics, University of Bergen, Bergen, Norway

petr.golovach@ii.uib.no

3 Department of Informatics, University of Bergen, Bergen, Norway

daniello@ii.uib.no

4 Department of Informatics, University of Bergen, Bergen, Norway; and Institute of Mathematical Sciences, Chennai, India

saket@imsc.res.in

\begin{abstract}
We consider 5the problem of covering a set of vectors of a given finite dimensional linear space (vector space) by a subspace generated by a set of vectors of minimum size. Specifically, we study the SPACE COver problem, where we are given a matrix $M$ and a subset of its columns $T$; the task is to find a minimum set $F$ of columns of $M$ disjoint with $T$ such that that the linear span of $F$ contains all vectors of $T$. This is a fundamental problem arising in different domains, such as coding theory, machine learning, and graph algorithms.

We give a parameterized algorithm with running time $2^{\mathcal{O}(k)} \cdot\|M\|^{\mathcal{O}(1)}$ solving this problem in the case when $M$ is a totally unimodular matrix over rationals, where $k$ is the size of $F$. In other words, we show that the problem is fixed-parameter tractable parameterized by the rank of the covering subspace. The algorithm is "asymptotically optimal" for the following reasons.

Choice of matrices: Vector matroids corresponding to totally unimodular matrices over rationals are exactly the regular matroids. It is known that for matrices corresponding to a more general class of matroids, namely, binary matroids, the problem becomes W[1]-hard being parameterized by $k$.

Choice of the parameter: The problem is NP-hard even if $|T|=3$ on matrix-representations of a subclass of regular matroids, namely cographic matroids. Thus for a stronger parameterization, like by the size of $T$, the problem becomes intractable.

Running Time: The exponential dependence in the running time of our algorithm cannot be asymptotically improved unless Exponential Time Hypothesis (ETH) fails.
\end{abstract}

Our algorithm exploits the classical decomposition theorem of Seymour for regular matroids.

1998 ACM Subject Classification G.2.2 Graph Theory, F.2.2 Nonnumerical Algorithms and Problems

Keywords and phrases regular matroids, spanning set, parameterized complexity

Digital Object Identifier 10.4230/LIPIcs.ICALP.2017.56 * The research leading to these results has received funding from the European Research Council under the
European Union's Seventh Framework Programme (FP/2007-2013) / ERC Grant Agreement n. 267959
and the Research Council of Norway via the projects "CLASSIS" and "MULTIVAL".

(c) (i) $\odot$ Fedor V. Fomin, Petr A. Golovach, Daniel Lokshtanov, and Saket Saurabh; censed under Creative Commons License CC-BY

44th International Colloquium on Automata, Languages, and Programming (ICALP 2017). Editors: Ioannis Chatzigiannakis, Piotr Indyk, Fabian Kuhn, and Anca Muscholl; Article No. 56; pp. 56:1-56:15

Leibniz International Proceedings in Informatics
LIPICS Schloss Dagstuhl - Leibniz-Zentrum für Informatik, Dagstuhl Publishing, Germany

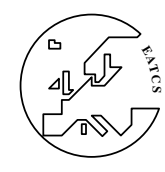
LIPICS Schloss Dagstuhl - Leibniz-Zentrum für Informatik, Dagstuhl Publishing, Germany 


\section{Introduction}

We consider the fundamental problem of covering a subspace of a given finite dimensional linear space (vector space) by a set of vectors of minimum size. The input of the problem is a matrix $M$ given together with a function $w$ assigning a nonnegative weight to each column of $M$ and a set $T$ of terminal column-vectors $T$ of $M$. The task is to find a minimum set of column-vectors $F$ of $M$ (if such a set exists) which is disjoint with $T$ and generates a subspace containing the linear space generated by $T$. In other words, $T \subseteq \operatorname{span}(F)$, where $\operatorname{span}(F)$ is the linear span of $F$. We refer to this problem as the SPACE Cover problem.

The SPACE COver problem encompasses various problems arising in different domains. The Minimum Distance problem in coding theory asks for a minimum dependent set of columns in a matrix over $\mathrm{GF}(2)$. This problem can be reduced to SpACE Cover by finding for each column $t$ in matrix $M$ a minimum set of columns in the remaining part of the matrix that cover $T=\{t\}$. The complexity of this problem was asked by Berlekamp et al. [2] and remained open for almost 30 years. It was resolved only in 1997, when Vardy showed it to be NP-complete [38]. The parameterized version of the Minimum Distance problem, namely Even SET, asks whether there is a dependent set $F \subseteq X$ of size at most $k$. The parameterized complexity of EVEN SET is a long-standing open question in the area, see e.g. [8]. In the language of matroid theory, the problem of finding a minimum dependent set is known as MATROID GiRTh, i.e. the problem of finding a circuit in matroid of minimum length [39]. In machine learning this problem is known as the SuBSPACE RECOVERY problem [20]. This problem also generalizes the problem of computing the rank of a tensor.

For our purposes, it is convenient to rephrase the definition of the SPACE Cover problem in the language of matroids. Given a matrix $N$, let $M=(E, \mathcal{I})$ denote the matroid where the ground set $E$ corresponds to the columns of $N$ and $\mathcal{I}$ denote the family of subsets of linearly independent columns. This matroid is called the vector matroid corresponding to matrix $N$. Then for matroids, finding a subspace covering $T$ corresponds to finding $F \subseteq E \backslash T, F \in \mathcal{I}$, such that $|F| \leq k$ and $T$ is spanned by $F$. Let us remind that in a matroid set $F$ spans $T$, denoted by $T \subseteq \operatorname{span}(F)$, if $r(F)=r(T \cup F)$. Here $r: 2^{E} \rightarrow \mathbb{N}_{0}$ is the rank function of $M$. (We use $\mathbb{N}_{0}$ to denote the set of nonnegative integers.)

Then SPACE COVER is defined as follows.

SPACE Cover

Parameter: $k$

Input: A binary matroid $M=(E, \mathcal{I})$ given together with its matrix representation over $\mathrm{GF}(2)$, a weight function $w: E \rightarrow \mathbb{N}_{0}$, a set of terminals $T \subseteq E$, and a nonnegative integer $k$.

Question: Is there a set $F \subseteq E \backslash T$ with $w(F) \leq k$ such that $T \subseteq \operatorname{span}(F)$ ?

Since a representation of a binary matroid is given as a part of the input, we always assume that the size of $M$ is $\|M\|=|E|$. For regular matroids, testing matroid regularity can be done efficiently, see e.g. [37], and when the input binary matroid is regular, the requirement that the matroid is given together with its representation can be omitted.

It is known (see, e.g., [26]) that SPACE COVER on special classes of binary matroids, namely graphic and cographic matroids, generalizes two well-studied optimization problems on graphs, namely Steiner Tree and Multiway CUT. Both problems play fundamental roles in parameterized algorithms.

Recall that in the STEINER FOREST problem we are given a (multi) graph $G$, a weight function $w: E \rightarrow \mathbb{N}$, a collection of pairs of distinct vertices $\left\{x_{1}, y_{1}\right\}, \ldots,\left\{x_{r}, y_{r}\right\}$ of $G$, and a nonnegative integer $k$. The task is to decide whether there is a set $F \subseteq E(G)$ with $w(F) \leq k$ 
such that for each $i \in\{1, \ldots, r\}$, graph $G[F]$ contains an $\left(x_{i}, y_{i}\right)$-path. To see that STEINER Forest is a special case of SpACE COver, for instance $\left(G, w,\left\{x_{1}, y_{1}\right\}, \ldots,\left\{x_{r}, y_{r}\right\}, k\right)$ of STEINER ForEST, we construct the following graph. For each $i \in\{1, \ldots, r\}$, we add a new edge $x_{i} y_{i}$ to $G$ and assign an arbitrary weight to it; notice that we can create multiple edges this way. Denote by $G^{\prime}$ the obtained mulitigraph and let $T$ be the set of added edges and let $M\left(G^{\prime}\right)$ be the graphic matroid associated with $G^{\prime}$. Then a set of edges $F \subseteq E(G)$ forms a graph containing all $\left(x_{i}, y_{i}\right)$-paths if an only if $T \subseteq \operatorname{span}(F)$ in $M\left(G^{\prime}\right)$.

The special case of STEINER FOREST when $x_{1}=x_{2}=\cdots=x_{r}$, i.e. when set $F$ should form a connected subgraph spanning all demand vertices, is the STEINER TREE problem, the fundamental problem in network optimization. By the classical result of Dreyfus and Wagner [10], SteINER TREE is fixed-parameter tractable (FPT) parameterized by the number of terminals. The study of parameterized algorithms for STEINER TREE has led to the design of important techniques, such as Fast Subset Convolution [3] and the use of branching walks [29]. Research on the parameterized complexity of STEINER TREE is still on-going, with recent significant advances for the planar version of the problem [33]. Algorithms for STEINER TREE are frequently used as a subroutine in FPT algorithms for other problems; examples include vertex cover problems [19], near-perfect phylogenetic tree reconstruction [4], and connectivity augmentation problems [1].

The dual of Space Cover, i.e., the variant of SpACE Cover asking whether there is a set $F \subseteq E \backslash T$ with $w(F) \leq k$ such that $T \subseteq \operatorname{span}(F)$ in the dual matroid $M^{*}$, is equivalent to the Restricted Subset FeEdBack Set problem. In this problem the task is for a given matroid $M$, a weight function $w: E \rightarrow \mathbb{N}_{0}$, and a nonnegative integer $k$, to decide whether there is a set $F \subseteq E \backslash T$ with $w(F) \leq k$ such that matroid $M^{\prime}$ obtained from $M$ by deleting the elements of $F$ has no circuit containing an element of $T$. Hence, SpACE Cover for cographic matroids is equivalent to Restricted Subset FeEdBack Set for graphic matroids. Restricted Subset Feedback Set for graphs was introduced by Xiao and Nagamochi [40], who showed that this problem is FPT parameterized by $|F|$. Let us note that in order to obtain an algorithm for SPACE COVER with a single-exponential dependence in $k$, we also need to design a new algorithm for SPACE COVER on cographic matroids which improves significantly the running time achieved by Xiao and Nagamochi [40].

Multiway CUt, another fundamental graph problem, is the special case of RESTRICTED Subset Feedback Set, and therefore of Space Cover. In the Multiway Cut problem we are given a (multi) graph $G$, a weight function $w: E \rightarrow \mathbb{N}$, a set $S \subseteq V(G)$, and a nonnegative integer $k$. The task is to decide whether there is a set $F \subseteq E(G)$ with $w(F) \leq k$ such that the vertices of $S$ are in distinct connected components of the graph obtained from $G$ by deleting edges of $F$. Indeed, let $(G, w, S, k)$ be an instance of Multiway Cut. We construct graph $G^{\prime}$ by adding a new vertex $u$ and connecting it to the vertices of $S$. Denote by $T$ the set of added edges and assign weights to them arbitrarily. Then $(G, w, S, k)$ is equivalent to the instance $\left(M\left(G^{\prime}\right), w, T, k\right)$ of Restricted Subset Feedback Set. If $|S|=2$, Multiway Cut is exactly the classical min-cut problem which is solvable in polynomial time. However, as it was proved by Dahlhaus et al. [6] already for three terminals the problem becomes NP-hard. Marx, in his celebrated work on important separators [28], has shown that Multiway CUT is FPT when parameterized by the size of the cut $|F|$.

While STEINER TREe is FPT parameterized by the number of terminal vertices, the hardness results for Multiway CUT with three terminals yields that SPACE Cover parameterized by the size of the terminal set $T$ is Para-NP-complete even if restricted to cographic matroids. This explains why we parameterize SPACE COVER by the rank of the span and not the size of the terminal set. 
There is also a strong argument that SPACE COvER is not tractable in its full generality on binary matroids for the following reason. It follows from the result of Downey et al. [9] on the hardness of the Maximum-Likelihood Decoding problem, that Space Cover is $\mathrm{W}[1]$-hard for binary matroids when parameterized by $k$ even if restricted to the inputs with one terminal and unit-weight elements. However, it is still possible to establish the tractability of the problem on a large class of binary matroids. Sandwiched between graphic and cographic (where the problem is FPT) and binary matroids (where the problem is intractable) is the class of regular matroids. Our main theorem establishes the tractability of SPACE COVER on regular matroids.

- Theorem 1. SPACE COVER on regular matroids is solvable in time $2^{\mathcal{O}(k)} \cdot\|M\|^{\mathcal{O}(1)}$.

We believe that due to the generality of SPACE COVER, Theorem 1 will be useful in the study of various optimization problems on regular matroids. As an example, we consider the RANK $h$-REDUCtion problem, see e.g. [24]. Here we are given a binary matroid $M$ and positive integers $h$ and $k$, the task is to decide whether it is possible to decrease the rank of $M$ by at least $h$ by deleting $k$ elements. For graphic matroids, this is the $h$-WAY Cut problem, which is for a connected graph $G$ and positive integers $h$ and $k$, to decide whether it is possible to separate $G$ into at least $h$ connected components by deleting at most $k$ edges. By the celebrated result of Kawarabayashi and Thorup [25], $h$-WAY CuT is FPT parameterized by $k$ even if $h$ is a part of the input. The result of Kawarabayashi and Thorup cannot be extended to cographic matroids; we show that for cographic matroids the problem is W[1]-hard when parameterized by $h+k$. On the other hand, by making use of Theorem 1, we solve RANK $h$-REDUCTION in time $2^{\mathcal{O}(k)} \cdot\|M\|^{\mathcal{O}(h)}$ on regular matroids.

Let us also remark that the running time of our algorithm is asymptotically optimal: unless Exponential Time Hypothesis fails, there is no algorithm of running time $2^{o(k)} \cdot\|M\|^{\mathcal{O}(1)}$ solving Space Cover on graphic (Steiner Tree) or cographic (Multiway Cut) matroids, see e.g. [5].

Related work. The main building block of our algorithm is the fundamental theorem of Seymour [34] on a decomposition of regular matroids. Roughly speaking (we define it properly in Section 2), the Seymour's decomposition provides a way to decompose a regular matroid into much simpler base matroids that are graphic, cographic or have a constant size in such way that all "communication" between base matroids is limited to "cuts" of small rank (we refer to the monograph of Truemper [37] and the survey of Seymour [35] for the introduction to matroid decompositions). This theorem has a number of important combinatorial and algorithmic applications. Among the classic algorithmic applications of Seymour's decomposition are the polynomial time algorithms of Truemper [36] (see also [37]) for finding maximum flows and shortest routes and the polynomial algorithm of Golynski and Horton [18] for constructing a minimum cycle basis. More recent applications of Seymour's decomposition can be found in approximation, on-line and parameterized algorithms. Godberg and Jerrum [17] used Seymour's decomposition theorem for obtaining a fully polynomial randomized approximation scheme (FPRAS) for the partition function of the ferromagnetic Ising model on regular matroids. Dinitz and Kortsarz in [7] applied the decomposition theorem for the Matroid Secretary problem. In [12], Gavenciak, Král and Oum initiated the study of the Minimum Spanning Circuit problem for matroids that generalizes the classical CyCle Through ElEments problem for graphs. The problem asks for a matroid $M$, a set $T \subseteq E$ and a nonnegative integer $\ell$, whether there is a circuit $C$ of $M$ with $T \subseteq C$ of size at most $\ell$. Gavenciak, Král and Oum [12] proved that the problem is 
FPT when parameterized by $\ell$ if $|T| \leq 2$. Very recently, in [11], we extended this result by showing that Minimum Spanning Circuit is FPT when parameterized by $k=\ell-|T|$.

On a very superficial level, all the algorithmic approaches based on the Seymour's decomposition theorem utilize the same idea: solve the problem on base matroids and then "glue" solutions into a global solution. However, such a view is a strong oversimplification. First of all, the original decomposition of Seymour in [34] was not meant for algorithmic purposes and almost every time to use it algorithmically one has to apply nontrivial adjustments to the original decomposition. For example, in order to solve MATROID SECRETARY on regular matroids, Dinitz and Kortsarz in [7] had to give a refined decomposition theorem suitable for their algorithmic needs. Similarly, in order to use the decomposition theorem for approximation algorithms, Goldberg and Jerrum in [17] had to add several new ingredients to the original Seymour's construction. We face exactly the same nature of difficulties in using Seymour's decomposition theorem. Our starting point is the variant of the decomposition theorem proved by Dinitz and Kortsarz in [7]. However, this theorem as it is can also not be used "statically" for our purposes. Our algorithm, while recursively constructing a solution has to "dynamically" transform the decomposition. This occurs when the algorithm processes cographic matroids "glued" with other matroids and for that part of the algorithm the transformation of the decomposition is essential.

\section{Algorithm roadmap}

In this section we give a high level overview of our algorithm for SPACE Cover. Due to space restrictions, all details and proofs are postponed for a journal version of our paper. We assume that the reader is acquainted with the basics of Matroid theory and refer to the book of Oxley [32] for the introduction.

We denote the ground set of matroid $M=(E, \mathcal{I})$ by $E(M)$ or simply by $E$ if it does not create confusion. Recall that a set $X \subseteq E$ spans $e \in E$ if $r(X \cup\{e\})=r(X)$, and $\operatorname{span}(X)=\{e \in E \mid X$ spans $e\}$, where $r$ is the rank function of $M$. Respectively, $X$ spans a set $T \subseteq E$ if $T \subseteq \operatorname{span}(X)$. An (inclusion) minimal dependent set is called a circuit of $M$. An one-element circuit is called loop, and if $\left\{e_{1}, e_{2}\right\}$ is a two-element circuit, then it is said that $e_{1}$ and $e_{2}$ are parallel. A set $X \subseteq E$ is a cycle of $M$ if $X$ is either empty or $X$ is a disjoint union of circuits. Let $G$ be a (multi) graph. The cycle matroid $M(G)$ has the ground set $E(G)$ and a set $X \subseteq E(G)$ is independent if $X=\emptyset$ or $G[X]$ has no cycles. Notice that $C$ is a circuit of $M(G)$ if and only if $C$ induces a cycle of $G$. The bond matroid $M^{*}(G)$ with the ground set $E(G)$ is dual to $M(G)$, and $X$ is a circuit of $M^{*}(G)$ if and only if $X$ is a minimal cut-set of $G$. It is said that $M$ is a graphic matroid if $M$ is isomorphic to $M(G)$ for some graph $G$ and $M$ is cographic if $M$ is isomorphic to $M^{*}(G)$.

Our algorithm uses the following observation.

- Observation 2. Let $e \in E$ and $X \subseteq E \backslash\{e\}$ for a matroid $M$. Then $e \in \operatorname{span}(X)$ if and only if there is a circuit $C$ such that $e \in C \subseteq X \cup\{e\}$.

By Observation 2, to solve Space Cover we have to find $F \subseteq E \backslash T$ with $w(F) \leq k$ such that for every $t \in T$, there is circuit $C$ of $M$ such that $t \in C \subseteq F \cup\{t\}$.

\subsection{Regular matroid decompositions}

In this section we describe matroid decomposition theorems that are pivotal for the algorithm for SPACE COver. Roughly speaking, the classical theorem of Seymour [34] says that every regular matroid can be decomposed via "small sums" into basic matroids which are graphic, 
cographic and very special matroid of constant size called $R_{10}$. To describe the decomposition of matroids, we need the notion of " $\ell$-sums" of matroids; we refer to $[32,37]$ for a formal introduction to matroid sums. However, for our purpose, it is sufficient that we restrict ourselves to binary matroids and up to 3-sums [34]. Recall that, for two sets $X$ and $Y$, $X \triangle Y=(X \backslash Y) \cup(Y \backslash X)$ denotes the symmetric difference of $X$ and $Y$. For two binary matroids $M_{1}$ and $M_{2}$, the sum of $M_{1}$ and $M_{2}$, denoted by $M_{1} \triangle M_{2}$, is the matroid $M$ with the ground set $E\left(M_{1}\right) \triangle E\left(M_{2}\right)$ whose cycles are all subsets $C \subseteq E\left(M_{1}\right) \triangle E\left(M_{2}\right)$ of the form $C=C_{1} \triangle C_{2}$, where $C_{1}$ is a cycle of $M_{1}$ and $C_{2}$ is a cycle of $M_{2}$.

- Definition 3 ( $\{1,2,3\}$-sum). For matroids $M_{1}, M_{2}$ and their sum $M$,

(S1) If $E\left(M_{1}\right) \cap E\left(M_{2}\right)=\emptyset$ and $E\left(M_{1}\right), E\left(M_{2}\right) \neq \emptyset$, then $M$ is the 1-sum of $M_{1}$ and $M_{2}$ and we write $M=M_{1} \oplus_{1} M_{2}$.

(S2) If $\left|E\left(M_{1}\right) \cap E\left(M_{2}\right)\right|=1$, then $M$ is the 2-sum of $M_{1}$ and $M_{2}$ and we write $M=$ $M_{1} \oplus_{2} M_{2}$.

(S3) If $\left|E\left(M_{1}\right) \cap E\left(M_{2}\right)\right|=3$, the 3-element set $Z=E\left(M_{1}\right) \cap E\left(M_{2}\right)$ is a circuit of $M_{1}$ and $M_{2}$, then $M$ is the 3-sum of $M_{1}$ and $M_{2}$ and we write $M=M_{1} \oplus_{3} M_{2}$.

If $M=M_{1} \oplus_{k} M_{2}$ for some $k \in\{1,2,3\}$, then we write $M=M_{1} \oplus M_{2}$.

Note that the definitions of (S2) and (S3) in [34] include some additional restrictions for $E\left(M_{1}\right) \cap E\left(M_{2}\right)$ but, as it was pointed by Dinitz and Kortsarz in [7], they are used only to ensure the nontriviality and can be omitted for algorithmic applications.

- Definition 4 (\{1,2,3\}-decomposition). A $\{1,2,3\}$-decomposition of a matroid $M$ is a collection of matroids $\mathcal{M}$, called the basic matroids, and a rooted binary tree $T$ in which $M$ is the root and the elements of $\mathcal{M}$ are the leaves such that any internal node is either 1-, 2or 3 -sum of its children.

We also need the special binary matroid $R_{10}$ which is represented over GF(2) by the $5 \times 10$-matrix whose columns are formed by vectors that have exactly three non-zero entries (or rather three ones) and no two columns are identical. Seymour's theorem [34] states that every regular matroid has a $\{1,2,3\}$-decomposition in which every basic matroid is graphic, cographic or isomorphic to $R_{10}$.

Dinitz and Kortsarz in [7] obtained a variant of matroid decomposition which is more handy for our purposes. This variant is based on the notion conflict graph.

- Definition $5([7])$. Let $(T, \mathcal{M})$ be a $\{1,2,3\}$-decomposition of a matroid $M$. The conflict (or intersection) graph of $(T, \mathcal{M})$ is the graph $G_{T}$ with the vertex set $\mathcal{M}$ such that distinct $M_{1}, M_{2} \in \mathcal{M}$ are adjacent in $G_{T}$ if and only if $E\left(M_{1}\right) \cap E\left(M_{2}\right) \neq \emptyset$.

- Theorem 6 ([7]). For a given regular matroid $M$, there is a (conflict) tree $\mathcal{T}$, whose set of nodes is a set of matroids $\mathcal{M}$, where each element of $\mathcal{M}$ is a graphic or cographic matroid, or a matroid obtained from $R_{10}$ by (possibly) adding parallel elements, that has the following properties: (i) if two distinct matroids $M_{1}, M_{2} \in \mathcal{M}$ have nonempty intersection, then $M_{1}$ and $M_{2}$ are adjacent in $\mathcal{T}$, (ii) for any distinct $M_{1}, M_{2} \in \mathcal{M}$, the intersection $E\left(M_{1}\right) \cap E\left(M_{2}\right)$ satisfies one of the properties (S1)-(S3) of 1, 2 or 3-sums, (iii) $M$ is obtained by the consecutive performing 1, 2 or 3-sums for adjacent matroids in any order. Moreover, $\mathcal{T}$ can be constructed in a polynomial time.

If $\mathcal{T}$ is a conflict tree for matroid $M$, we say that $M$ is defined by $\mathcal{T}$. 


\subsection{Elementary reductions for Space Cover}

In this section we give some elementary reduction rules that we apply on the instances of SPACE COVER to make it more structured. This structure will be exploited by our FPT algorithm. In particular, our algorithm crucially utilizes the fact that the solution $F$ we are seeking is of size at most $k$. However, the way our algorithm is designed, in certain cases the weights of elements can be changed and it can occur that some elements could have been assigned weight zero by $w$. In this case a solution $F$ of weight at most $k$ does not imply that it is a solution of size at most $k$. These reduction rules allow us to take care of such situations.

- Reduction Rule 1 (Zero-element). If there is an element $e \in E \backslash T$ with $w(e)=0$, then contract e.

Reduction Rule 2 (Terminal circuit). If there is a circuit $C \subseteq T$, then delete an arbitrary element $e \in C$ from $M$.

Let us note that Reduction Rules 1 and 2 can be applied in time polynomial in $\|M\|$.

\subsection{Solving Space Cover for basic matroids}

We start by solving SPACE COVER on basic matroids that are building blocks of regular matroid: $R_{10}$, graphic and cographic matroids. For $R_{10}$ the solution is trivial and for graphic matroids it is an easy extension of the classic Dreyfus-Wagner algorithm [10] for STEINER TREE. However, a single-exponential algorithm for cographic matroids requires new ideas.

Thus we obtain the following lemmata.

- Lemma 7. Space COVER can be solved in polynomial time for matroids that can be obtained from $R_{10}$ by adding parallel elements, element deletions and contractions.

- Lemma 8. SPACE COVER on graphic matroids is solvable in time $4^{k} \cdot\|M\|^{\mathcal{O}(1)}$.

- Lemma 9. SPACE COVER can be solved in time $2^{\mathcal{O}(k)} \cdot\|M\|^{\mathcal{O}(1)}$ on cographic matroids.

By the results of Xiao and Nagamochi [40], Restricted Subset Feedback Set can be solved in time $2^{\mathcal{O}(k \log k)} \cdot\|M\|^{\mathcal{O}(1)}$ on graphic matroids. It immediately implies that Space Cover can be solved in the same time on cographic matroids by the duality of these problems. To improve this running time and get a single-exponential dependence in $k$, we construct a new algorithm based on the idea of enumeration of important cuts proposed by Marx in [28], see also [5]. Let $G$ be a graph such that $M$ is isomorphic to the bond matroid $M^{*}(G)$ of $G$. By the duality of Space Cover and Restricted Subset Feedback Set, a set $F \subseteq E(G) \backslash T$ spans $T$ if and only if the edges of $T$ are the bridges of $G-F$. The set of circuits of $M$ is the set of inclusion-minimal edge cut-sets of $G$. Hence we restate SpACE Cover as a cut problem in $G$ : for a given set $T \subseteq E(G)$, we need to find a minimum set $F \subseteq E(G) \backslash T$ such that the edges of $T$ are bridges of $G-F$. For our purpose, we need to modify the definition of an important cut given by Marx [28,5]. Let $s \in V(G)$ be a vertex of $G, T \subseteq V(G) \backslash\{s\}$ be a set of terminals, and $k$ be a nonnegative integer. We say that a set $W \subseteq V(G)$ is interesting if $G[W]$ is connected, $s \in W$, and $|T \cap W| \leq 1$. For $W \subseteq V(G)$, by $\Delta(W)$ we denote the set of edges of $G$ with exactly one end-vertex in $W$. Given two interesting sets $W_{1}$ and $W_{2}$ we say that $W_{1}$ is better than $W_{2}$ and denote by $W_{2} \preceq W_{1}$ if $W_{2} \subseteq W_{1},\left|\Delta\left(W_{1}\right)\right| \leq\left|\Delta\left(W_{2}\right)\right|$, and $T \cap W_{1} \subseteq T \cap W_{2}$. For set of terminals $T \subseteq V(G) \backslash\{s\}$, an interesting set $W$ is $(s, T, k)$-semi-important if $|\Delta(W)| \leq k$ and there is 
no set $W^{\prime}$ such that $W \preceq W^{\prime}$. The set of edges $\Delta(W)$ of a $(s, T, k)$-semi-important set $W$ is called a $(s, T, k)$-semi-important cut. We show that the number of $(s, T, k)$-semi-important cuts is in $16^{k} \cdot n^{\mathcal{O}(1)}$. Moreover, such cuts can be enumerated within the same time. The crux in the proof of Lemma 9 is the recursive algorithm computing the solution. The running time of the algorithm can be estimated by a polynomial of the number of $(s, T, k)$-semi-important cuts.

\subsection{Solving Space Cover for regular matroids}

Now we conjure all that have developed so far and design an algorithm for SPACE COVER on regular matroids, running in time $2^{\mathcal{O}(k)} \cdot\|M\|^{\mathcal{O}(1)}$. We first give some generic steps, followed by steps when matroid in consideration is either graphic and cographic and ending with a result that ties them all.

Let $(M, w, T, k)$ be a given instance of SPACE Cover. First, we exhaustively apply Reduction Rules 1-2. To simplify notations, we also denote the reduced instance by $(M, w, T, k)$. We say that a matroid $M$ is basic if it is graphic, cographic or can be obtained from $R_{10}$ by adding parallel elements. By Lemmata 7, 8, and 9, we have the following lemma.

- Lemma 10. Let $(M, w, T, k)$ be an instance of SpaCE COVER. If $M$ is a basic matroid, then SPACE COVER can be solved in time $2^{\mathcal{O}(k)} \cdot\|M\|^{\mathcal{O}(1)}$.

From now onwards we assume that matroid $M$ in the instance $(M, w, T, k)$ is not basic. Now using Theorem 6 , we find a conflict tree $\mathcal{T}$. Recall that the set of nodes of $\mathcal{T}$ is the collection of basic matroids $\mathcal{M}$, its the edges correspond to 1-, 2- and 3-sums and that $M$ can be constructed from $\mathcal{M}$ by performing the sums corresponding to the edges of $\mathcal{T}$ in an arbitrary order. Our algorithm is based on performing bottom-up traversal of the tree $\mathcal{T}$. We select an arbitrarily node $r$ as the root of $\mathcal{T}$. This defines the natural parent-child relationship for the nodes of $\mathcal{T}$. We say that node $M_{s}$ is a sub-leaf if all its children are leaves of $\mathcal{T}$. Observe that there always exists a sub-leaf in a tree on at least two nodes and that this node can be found in polynomial time.

We first modify the decomposition by an exhaustive application of the following rule.

- Reduction Rule 3 (Terminal flipping). If there is a child $M_{\ell}$ of a sub-leaf $M_{s}$ such that there is $e \in E\left(M_{s}\right) \cap E\left(M_{\ell}\right)$ that is parallel to a terminal $t \in E\left(M_{\ell}\right) \cap T$ in $M_{\ell}$, then delete $t$ from $M_{\ell}$ and add $t$ to $M_{s}$ as an element parallel to $e$.

It is easy to show that Reduction Rule 3 is safe and can be applied in polynomial time. From now we assume that there is no child $M_{\ell}$ of $M_{s}$ such that there exists an element $e \in E\left(M_{s}\right) \cap E\left(M_{\ell}\right)$ that is parallel to a terminal $t \in E\left(M_{\ell}\right) \cap T$ in $M_{\ell}$. This is important because it allows us to reduce the parameter while branching. In what follows, we do a bottom-up traversal of $\mathcal{T}$ and at each step we delete one of the children of $M_{s}$. A child of $M_{s}$ is deleted either because of an application of a reduction rule, or because of recursively solving the problem on a smaller sized tree. It is possible that, while recursively solving the problem, we could possibly modify (or replace) $M_{s}$ to encode some auxiliary information that we have already computed while solving the problem. If at some moment we arrive at the case $T=\emptyset$, then algorithm returns yes and stops. If at some moment the situation with $E \backslash T=\emptyset$ or $|T|>k$ occurs, then we return no and stop.

\subsubsection{Processing leaves}

For a sub-leaf node $M_{s}$, we say that a child $M_{\ell}$ of $M_{s}$ is a 1,2 or 3-leaf if the edge between $M_{s}$ and $M_{\ell}$ corresponds to 1, 2 or 3 -sum respectively. While the cases with 1 - and 2-leaves 
are relatively easy to settle, the case when $M_{\ell}$ is a 3 -leaf is difficult. We start from generic steps which do not depend on the types of $M_{s}$ and its child.

If $M_{\ell}$ is an 1-leaf such that $E\left(M_{\ell}\right)$ does not contain terminals, we simply delete $M_{\ell}$ from $\mathcal{T}$ and consider the problem for the matroid defined by the obtained tree. If $M_{\ell}$ is an 1-leaf such that $T_{\ell}=E\left(M_{\ell}\right) \cap T \neq \emptyset$, then we can solve SPACE COVER for $M_{\ell}$ with the set of terminals $T_{\ell}$ independently. More formally, we find minimum $k^{\prime} \leq k$ such that $\left(M_{\ell}, w_{\ell}, T_{\ell}, k^{\prime}\right)$ is a yes-instance of SPACE COVER using Lemma 10. Then if such $k^{\prime}$ exist, we consider the matroid $M^{\prime}$ defined by $\mathcal{T}^{\prime}$ obtained from $\mathcal{T}$ by the deletion of $M_{s}$ and then solve the problem for $\left(M^{\prime}, w, T \cap E\left(M^{\prime}\right), k-k^{\prime}\right)$. Safeness of this reduction immediately follows from the definition of 1 -sum, and the reduction can be done in time $2^{\mathcal{O}(k)} \cdot\|M\|^{\mathcal{O}(1)}$.

For 2-leaves, we either reduce a leaf or apply a recursive procedure based on whether the leaf contains a terminal or not. Let $M_{\ell}$ be 2-leaf that is adjacent to $M_{s}$ is $\mathcal{T}$ and $E\left(M_{s}\right) \cap E\left(M_{\ell}\right)=\{e\}$. Let also $M^{\prime}$ be the matroid defined by $\mathcal{T}^{\prime}$ obtained from $\mathcal{T}$ by the deletion of $M_{\ell}$.

If $M_{\ell}$ does not contain terminals, we find a circuit of $M_{\ell}$ of minimum weight $w_{\ell}$ containing $e$ assuming that the weight of $e$ is 0 . Notice, that this can be done in time $2^{\mathcal{O}(k)} \cdot\|M\|^{\mathcal{O}(1)}$ by solving Space COVER on $M_{\ell}$ for the unique terminal $e$. Then we delete $M_{\ell}$ from $\mathcal{T}$, assign the weight $w_{\ell}$ to the element $e$ of $M_{s}$ and then solve the problem for $M^{\prime}$.

Suppose that $M_{\ell}$ is a 2-leaf with terminals. Let $T_{\ell}=E\left(M_{\ell}\right) \cap T$ and $T^{\prime}=T \backslash T_{\ell}$. Notice that due to Reduction Rule $3, M_{\ell}$ has no terminal parallel to $e$. In particular, it can be shown that this implies that the total weight of the elements of $M_{\ell}$ in any solution is positive and this makes the branching possible, because the selection of elements of a solution in $M_{\ell}$ reduces the parameter. Notice here that we allow zero weights but all such nonterminal elements are contracted by Reduction Rule 1 . We have three branching cases corresponding to the behavior of a (potential) solution $F$. Recall that by Observation 2 , for each $t \in T$, there is a circuit $C$ such that $t \in C \subseteq F \cup\{t\}$.

Case 1. There is $t \in T^{\prime}$ and a circuit $C$ of $M$ such that $t \in C \subseteq F \cup\{t\}$ and $C$ contains an element of $M_{\ell}$. To handle this case, we consider SPACE COVER on $M_{\ell}$ with the terminals $T_{e} \cup\{e\}$, that is, we declare $e$ to be a terminal. We find the minimum $0<k^{\prime} \leq k$ such that $\left(M_{\ell}, w, T_{\ell} \cup\{e\}, k^{\prime}\right)$ is a yes-instance of SPACE COVER using Lemma 10. Then we assign the weight 0 to $e$ in $M_{s}$ and solve the problem for $\left(M^{\prime}, w, T^{\prime}, k-k^{\prime}\right)$.

Case 2. There is $t \in T_{\ell}$ and a circuit $C$ of $M$ such that $t \in C \subseteq F \cup\{t\}$ and $C$ contains an element of $M^{\prime}$. This case is handled symmetrically to Case 1 and, respectively, we find the minimum $0<k^{\prime} \leq k$ such that $\left(M_{\ell}, w, T_{\ell}, k^{\prime}\right)$ is a yes-instance of SPACE COVER where $e$ is assumed to have the weight 0 . Then we solve the problem for $\left(M^{\prime}, w, T^{\prime} \cup\{e\}, k-k^{\prime}\right)$.

Case 3. None of the above cases occur, i.e., every terminal from $M_{\ell}$ is spanned by elements of $M_{\ell}$ in $F$ and every terminal from $M^{\prime}$ is spanned by elements of $M^{\prime}$. Then we can solve the problem independently for $M_{\ell}$ and $M^{\prime}$ assuming that the weight of $e$ is $k+1$ which forbids using $e$ in a solution. We find minimum $0<k^{\prime} \leq k$ such that $\left(M_{\ell}, w_{\ell}, T_{\ell}, k^{\prime}\right)$ is a yes-instance of SPACE COver and then solve the problem for $\left(M^{\prime}, w, T^{\prime}, k-k^{\prime}\right)$.

It is possible to show this branching is exhaustive. We show also that one call of the step (without recursive calls) can be done in time $2^{\mathcal{O}(k)} \cdot\|M\|^{\mathcal{O}(1)}$.

Suppose now that $M_{\ell}$ is a 3-leaf adjacent to $M_{s}$ in $\mathcal{T}$. We again differentiate between cases when it has terminals or not. Assume that $M_{\ell}$ contains $T_{\ell}=E\left(M_{\ell}\right) \cap T \neq \emptyset$. Denote 
by $Z=E\left(M_{s}\right) \cap E\left(M_{\ell}\right)$. As for 2-leaves, we observe that there are no terminals of $T_{\ell}$ that are parallel to the elements of $Z$ in $M_{\ell}$ and we can branch according to the possible variants of the behavior of a (potential) solution $F$. The case analysis is technically complicated, because we have more variants of the behavior of $F$ comparing to the case analysis for 2-leaves. Still, the majority of the cases are handled by similar arguments and we omit them here, but there is one case that makes our algorithm complicated and we briefly discuss it here.

Denote by $M^{\prime}$ the matroid defined by $\mathcal{T}^{\prime}$ obtained from $\mathcal{T}$ by the deletion of $M_{\ell}$. Suppose that there is $t \in T^{\prime}$ and a circuit $C$ of $M$ such that $t \in C \subseteq F \cup\{t\}$ and $C$ contains an element of $M_{\ell}$, and there is $t^{\prime} \in T_{\ell}$ and a circuit $C^{\prime}$ of $M$ such that $t^{\prime} \in C^{\prime} \subseteq F \cup\left\{t^{\prime}\right\}$ and $C^{\prime}$ contains an element of $M^{\prime}$. Then it can be shown that there are distinct $e_{i}, e_{j} \in Z$ such that $C=C_{1} \triangle C_{2}, C^{\prime}=C_{1}^{\prime} \triangle C_{2}^{\prime}$ where $C_{1}, C_{1}^{\prime}$ are circuits of $M_{\ell}, C_{2}, C_{2}^{\prime}$ are circuits of $M^{\prime}, C_{1} \cap C_{2}=\left\{e_{i}\right\}$ and $C_{1}^{\prime} \cap C_{2}^{\prime}=\left\{e_{j}\right\}$. We declare $w\left(e_{i}\right)=w\left(e_{j}\right)=0$ and let the weight of the third element of $Z$ to be $k+1$. Then we find the minimum $0<k^{\prime} \leq k$ such that $\left(M_{\ell}, w, T_{\ell} \cup\left\{e_{i}\right\}, k^{\prime}\right)$ is a yes-instance of SPACE COvER and afterwards solve the problem for $\left(M^{\prime}, w, T^{\prime} \cup\left\{e_{j}\right\}, k-k^{\prime}\right)$.

However, it can lead to the following situation. We have solutions $F_{\ell}$ and $F^{\prime}$ for $\left(M_{\ell}, w, T_{\ell} \cup\left\{e_{i}\right\}, k^{\prime}\right)$ and $\left(M^{\prime}, w, T^{\prime} \cup\left\{e_{j}\right\}, k-k^{\prime}\right)$. Then there are circuits $C_{\ell}$ of $M_{\ell}$ and $C^{\prime}$ of $M^{\prime}$ such that $e_{i} \in C_{\ell} \subseteq F_{\ell} \cup\left\{e_{i}\right\}$ and $e_{j} \in C^{\prime} \subseteq F^{\prime} \cup\left\{e_{j}\right\}$. If $e_{j} \in C_{\ell}$ and $e_{i} \in C^{\prime}$, then $\left(F_{\ell} \triangle F^{\prime}\right) \backslash Z$ is not a solution for $M$. To avoid this situation, we have to solve a special variant of SPACE COVER for $\left(M_{\ell}, w, T_{\ell} \cup\left\{e_{i}\right\}, k^{\prime}\right)$ where we put on the solution $F_{\ell}$ the additional condition that $e_{i} \in \operatorname{span}\left(F_{\ell} \backslash e_{j}\right)$. Due to this technicality, we also have to provide algorithms solving this version of SPACE COVER on basic matroids. This is done by constructing variants of the algorithm from Lemmata 8 and 9.

For this branching, we show that it is exhaustive and one call of this branching step (without recursive calls) can be done in time $2^{\mathcal{O}(k)} \cdot\|M\|^{\mathcal{O}(1)}$.

We approach the most challenging part concerning processing of 3-leaves without terminals. At this stage we can assume that $\mathcal{T}$ has only 3 -leaves. The way to handle this case depends on the type of the sub-leaf $M_{s}$ adjacent to a 3-leaf $M_{\ell}$. Since $M_{\ell}$ is a 3-leaf, we have that $M_{s}$ is either graphic or cographic, because $R_{10}$ has no circuit of odd size.

Suppose that $M_{s}$ is a graphic matroid. Let $G$ be a graph such that its cycle matroid $M(G)$ is isomorphic to $M_{s}$. The algorithm that constructs a good $\{1,2,3\}$-decomposition also could be used to output the graph $G$. We assume that $M(G)=M_{s}$. The idea is to replace $M_{\ell}$ by attaching a gadget to $G$. Recall that the circuits of $M(G)$ are exactly the cycles of $G$. Since $Z$ is a circuit of $M(G)$, the elements of $Z$ form a cycle of $G$. Denote by $v_{1}, v_{2}, v_{3}$ its vertices. We modify $G$ by adding a new vertex $u$ and making it adjacent to $v_{1}, v_{2}, v_{3}$. Denote by $G^{\prime}$ the obtained graph. We assign weights to the edges of $Z$ and the new edges according to the possible structure of a solution $F$ in $M_{\ell}$ by solving auxiliary instances of Space Cover on $M_{\ell}$. It can happen that to span $T$ in $M$, we only need the property that $F \cap E\left(M_{\ell}\right)$ spans in $M_{\ell}$ a unique edge $v_{i} v_{j}$ of $Z$. Then we find a spanning set $F_{\ell}$ of minimum weight $w_{i j} \leq k$ in $M_{\ell}$ for the terminal $v_{i} v_{j}$ such that $v_{h} v_{i}, v_{h} v_{j} \notin F_{\ell}$ for $h \neq i, j$. Then we define $w\left(v_{i} v_{j}\right)=w_{i j}$ if $F_{\ell}$ exists and set $w\left(v_{i} v_{j}\right)=k+1$ otherwise. The other important possibility is that $F \cap E\left(M_{\ell}\right)$ spans in $M_{\ell}$ all $v_{i} v_{j}$ for distinct $i, j \in\{1,2,3\}$. We find a spanning set $F_{\ell}$ of minimum weight $w^{\prime} \leq k$ in $M_{\ell}$ for the terminal set $Z$. We show that it is possible to assign the weights to the edges $u v_{i}$ for $i \in\{1,2,3\}$ in such a way that $w\left(u v_{1}\right)+w\left(u v_{2}\right)+w\left(u v_{3}\right)=w^{\prime}$ and $w\left(u v_{i}\right)+w\left(u v_{j}\right) \geq w\left(v_{i} v_{j}\right)$ for $i, j \in\{1,2,3\}$ if such a solution $F_{\ell}$ exist. Otherwise, we simply set $w\left(u v_{i}\right)=k+1$ for $i \in\{1,2,3\}$. To complete the reduction, we consider the matroid $M^{\prime}$ defined by $\mathcal{T}^{\prime}$ obtained from $\mathcal{T}$ by the deletion of $M_{\ell}$ where $M_{s}$ is replaced by $M\left(G^{\prime}\right)$. Then we solve Space Cover for $\left(M^{\prime}, w, T, k\right)$. We show that the reduction can be done in time $2^{\mathcal{O}(k)} \cdot\|M\|^{\mathcal{O}(1)}$. 
The case when $M_{s}$ is a cographic matroid is most challenging. Let $G$ be a graph such that the bond matroid of $G$ is isomorphic to $M_{s}$. Without loss of generality, we can assume that $G$ is connected. Recall also that the circuits of the bond matroid $M^{*}(G)$ are exactly minimal cut-sets of $G$.

Isomorphism between $M_{s}$ and $M^{*}(G)$ is not necessarily unique. We choose an isomorphism between $M_{s}$ and $M^{*}(G)$ that is beneficial for our algorithmic purposes. Let $M_{\ell}^{(1)}, \ldots, M_{\ell}^{(s)}$ denote those leaves of the conflict tree $\mathcal{T}$ that are also the children of $M_{s}$. Let $Z_{i}=$ $E\left(M_{s}\right) \cap E\left(M_{\ell}^{(i)}\right), i \in\{1, \ldots, s\}$. If $M_{s}$ has a parent $M^{*}$ in $\mathcal{T}$ and $E\left(M_{s}\right) \cap E\left(M^{*}\right) \neq \emptyset$, then let $Z^{*}=E\left(M_{s}\right) \cap E\left(M^{*}\right)$; we emphasize that $Z^{*}$ may not exist. Next we define the notion of a clean cut.

- Definition 11. We say that $\alpha\left(Z_{i}\right) \subseteq E(G)$ is a clean cut with respect to an isomorphism $\alpha: M_{s} \rightarrow M^{*}(G)$, if there is a component $H$ of $G-\alpha\left(Z_{i}\right)$ such that (i) $H$ has no bridge, (ii) $E(H) \cap \alpha\left(Z_{j}\right)=\emptyset$ for $j \in\{1, \ldots, s\}$, and (iii) $E(H) \cap \alpha\left(Z^{*}\right)=\emptyset$ if $Z^{*}$ exists. We call $H$ a clean component of $G-\alpha\left(Z_{i}\right)$.

Next we show that given any isomorphism between $M_{s}$ and $M^{*}(G)$, we can obtain another isomorphism between $M_{s}$ and $M^{*}(G)$ with respect to which we have at least one clean component.

- Lemma 12. There is an isomorphism $\alpha: M_{s} \rightarrow M^{*}(G)$ and a child $M_{\ell}^{(i)}$ of $M_{s}$ such that $\alpha\left(Z_{i}\right)$ is a clean cut with respect to $\alpha$. Moreover, given any arbitrary isomorphism from $M_{s}$ to $M^{*}(G)$, one can obtain such an isomorphism and a clean cut together with a clean component in polynomial time.

Using Lemma 12, we can always assume that we have an isomorphism of $M_{s}$ to $M^{*}(G)$ such that for a child $M_{\ell}$ of $M_{s}$ in $\mathcal{T}, Z=E\left(M_{s}\right) \cap E\left(M_{\ell}\right)$ is mapped to a clean cut. To simplify notations, we assume that $M_{s}=M^{*}(G)$ and $Z$ is a clean cut with respect to this isomorphism. Denote by $H$ the clean component. Let $Z=\left\{e_{1}, e_{2}, e_{3}\right\}$ and let $e_{i}=x_{i} y_{i}$ for $i \in\{1,2,3\}$, where $y_{1}, y_{2}, y_{3} \in V(H)$. Notice that some $y_{1}, y_{2}, y_{3}$ can be the same.

We first handle the case when $E(H) \cap T=\emptyset$. Similarly to the case of a graphic subleaf, we replace $M_{\ell}$ by a gadget. The difference is that the gadget replaces $M_{s}$ and $H$. We modify $G$ as follows. First, we delete $H$. Then we construct three new pairwise adjacent vertices $z_{1}, z_{2}, z_{3}$ and make $z_{i}$ adjacent to $x_{i}$ for $i \in\{1,2,3\}$. Let $G^{\prime}$ be the obtained graph. We analyze the possible structure of a solution in $H$ and $M_{\ell}$, and use this information to assign weight to the edges $x_{i} z_{i}$ for $i \in\{1,2,3\}$ and $z_{i} z_{j}$ for $i, j \in\{1,2,3\}$ similarly to the case of a graphic subleaf. Finally, we consider the matroid $M^{\prime}$ defined by $\mathcal{T}^{\prime}$ obtained from $\mathcal{T}$ by the deletion of $M_{s}$ where $M_{s}$ is replaced by $M\left(G^{\prime}\right)$. Then we solve SPACE Cover for $\left(M^{\prime}, w, T, k\right)$. We prove that the reduction can be done in time $2^{\mathcal{O}(k)} \cdot\|M\|^{\mathcal{O}(1)}$.

It remains to consider the case $E(H) \cap T \neq \emptyset$. In this case, we either reduce $H$ or recursively solve the problem on smaller $H$. Rather than describing these steps, we observe that we can decompose $M_{s}$ further using the decomposition theorem given in [37, Chapter $8]$ using the cut $\left\{x_{1} y_{1}, x_{2} y_{2}, x_{3} y_{3}\right\}$. This way, we obtain a new leaf with terminals and can apply the already described rules.

Concerning the total running time, observe that we apply reduction rules either in polynomial time or in $2^{\mathcal{O}(k)} \cdot\|M\|^{\mathcal{O}(1)}$ time. After each reduction rule we obtain a conflict tree with a smaller number of vertices, hence we use reductions a polynomial number of times. For each of the branching rule, in the recursive call we reduce the parameter, hence the number of nodes in the corresponding search tree is in $2^{\mathcal{O}(k)}$. Therefore the running time of the algorithm is $2^{\mathcal{O}(k)} \cdot\|M\|^{\mathcal{O}(1)}$. 


\section{Reducing rank}

In the well-known $h$-WAY CUT problem, we are given a connected graph $G$ and positive integers $h$ and $k$, the task is to find at most $k$ edges whose removal increases the number of connected components by at least $h$. The problem has a simple formulation in terms of matroids: Given a graph $G$ and an integers $k, h$, find $k$ elements of the graphical matroid of $G$ whose removal reduces its rank by at least $h$. This motivated Joret and Vetta [24] to introduce the RANK $h$-REDUCTION problem on matroids. Here we define RANK $h$-REDUCTION on binary matroids.

RANK $h$-REDUCTION

Parameter: $k$

Input: A binary matroid $M=(E, \mathcal{I})$ given together with its matrix representation over $\mathrm{GF}(2)$ and two positive integers $h$ and $k$.

Question: Is there a set $X \subseteq E$ with $|X| \leq k$ such that $r(M)-r(M-X) \geq h$ ?

As a corollary of Theorem 1 , we show that on regular matroids RANK $h$-REDUCTION is FPT for any fixed $h$.

We use the following lemma.

- Lemma 13. Let $M$ be a binary matroid and let $k \geq h$ be positive integers. Then $M$ has a set $X \subseteq E$ with $|X| \leq k$ such that $r(M)-r(M-X) \geq h$ if and only if there are disjoint sets $F, T \subseteq E$ such that $|T|=h,|F| \leq k-h$, and $T \subseteq \operatorname{span}(F)$ in $M^{*}$.

For graphic matroids, when RANK $h$-REDUCTION is equivalent to $h$-WAY CUT, the problem is FPT parameterized by $k$ even if $h$ is a part of the input [25]. Unfortunately, similar result does not hold for cographic matroids.

- Proposition 14. RANK $h$-REDUCTION is W[1]-hard for cographic matroids parameterized by $h+k$.

However, by Theorem 1 , for fixed $h$, RANK $h$-REDUCTION is FPT parameterized by $k$ on regular matroids.

- Theorem 15. RANK $h$-REDUCTION can be solved in time $2^{\mathcal{O}(k)} \cdot\|M\|^{\mathcal{O}(h)}$ on regular matroids.

\section{Conclusion}

In this paper, we used the structural theorem of Seymour for designing parameterized algorithm for SPACE COVER. While structural graph theory and graph decompositions serve as the most usable tools in the design of parameterized algorithms, the applications of structural matroid theory in parameterized algorithms are limited. There is a series of papers about width-measures and decompositions of matroids (see, in articular, [21, 22, 23, 27, 30, 31] and the bibliography therein) but, apart of this specific area, we are not aware of other applications except the works Gavenciak et al. [12] and our recent work [11]. In spite of the tremendous progress in understanding the structure of matroids representable over finite fields $[13,14,15,16]$, this rich research area still remains to be explored from the perspective of parameterized complexity.

As a concrete open problem, what about the parameterized complexity of SPACE COVER on any proper minor-closed class of binary matroids? 


\section{References}

1 Manu Basavaraju, Fedor V. Fomin, Petr A. Golovach, Pranabendu Misra, M. S. Ramanujan, and Saket Saurabh. Parameterized algorithms to preserve connectivity. In Automata, Languages, and Programming - 41st International Colloquium, ICALP 2014, Copenhagen, Denmark, July 8-11, 2014, Proceedings, Part I, volume 8572 of Lecture Notes in Computer Science, pages 800-811. Springer, 2014. doi:10.1007/978-3-662-43948-7_66.

2 Elwyn R. Berlekamp, Robert J. McEliece, and Henk C. A. van Tilborg. On the inherent intractability of certain coding problems (corresp.). IEEE Trans. Information Theory, 24(3):384-386, 1978. doi:10.1109/TIT.1978.1055873.

3 Andreas Björklund, Thore Husfeldt, Petteri Kaski, and Mikko Koivisto. Fourier meets möbius: fast subset convolution. In Proceedings of the 39th Annual ACM Symposium on Theory of Computing, San Diego, California, USA, June 11-13, 2007, pages 67-74. ACM, 2007.

4 Guy E. Blelloch, Kedar Dhamdhere, Eran Halperin, R. Ravi, Russell Schwartz, and Srinath Sridhar. Fixed parameter tractability of binary near-perfect phylogenetic tree reconstruction. In Automata, Languages and Programming, 33rd International Colloquium, ICALP 2006, Venice, Italy, July 10-14, 2006, Proceedings, Part I, volume 4051 of Lecture Notes in Computer Science, pages 667-678. Springer, 2006. doi:10.1007/11786986_58.

5 Marek Cygan, Fedor V. Fomin, Lukasz Kowalik, Daniel Lokshtanov, Dániel Marx, Marcin Pilipczuk, Michal Pilipczuk, and Saket Saurabh. Parameterized Algorithms. Springer, 2015. doi: 10.1007/978-3-319-21275-3.

6 Elias Dahlhaus, David S. Johnson, Christos H. Papadimitriou, Paul D. Seymour, and Mihalis Yannakakis. The complexity of multiterminal cuts. SIAM J. Comput., 23(4):864894, 1994. doi:10.1137/S0097539792225297.

7 Michael Dinitz and Guy Kortsarz. Matroid secretary for regular and decomposable matroids. SIAM J. Comput., 43(5):1807-1830, 2014. doi:10.1137/13094030X.

8 Rodney G. Downey and Michael R. Fellows. Fundamentals of Parameterized Complexity. Texts in Computer Science. Springer, 2013. doi :10.1007/978-1-4471-5559-1.

9 Rodney G. Downey, Michael R. Fellows, Alexander Vardy, and Geoff Whittle. The parametrized complexity of some fundamental problems in coding theory. SIAM J. Comput., 29(2):545-570, 1999. doi:10.1137/s0097539797323571.

10 S. E. Dreyfus and R. A. Wagner. The Steiner problem in graphs. Networks, 1(3):195-207, 1971. doi:10.1002/net.3230010302.

11 Fedor V. Fomin, Petr A. Golovach, Daniel Lokshtanov, and Saket Saurabh. Spanning circuits in regular matroids. In Proceedings of the Twenty-Eighth Annual ACM-SIAM Symposium on Discrete Algorithms, SODA 2017, pages 1433-1441. SIAM, 2017.

12 Tomás Gavenciak, Daniel Král, and Sang-il Oum. Deciding first order properties of matroids. In Automata, Languages, and Programming - 39th International Colloquium, ICALP 2012, Warwick, UK, July 9-13, 2012, Proceedings, Part II, volume 7392, pages 239-250. Springer, 2012.

13 James F. Geelen, A.M.H. Gerards, and Geoff Whittle. Branch-width and well-quasiordering in matroids and graphs. J. Comb. Theory, Ser. B, 84(2):270-290, 2002. doi: 10.1006/jctb. 2001.2082.

14 Jim Geelen, Bert Gerards, and Geoff Whittle. Excluding a planar graph from gf(q)representable matroids. J. Comb. Theory, Ser. B, 97(6):971-998, 2007. doi:10.1016/ j.jctb.2007.02.005.

15 Jim Geelen, Bert Gerards, and Geoff Whittle. Solving Rota's conjecture. Notices Amer. Math. Soc., 61(7):736-743, 2014. doi:10.1090/noti1139.

16 Jim Geelen, Bert Gerards, and Geoff Whittle. The Highly Connected Matroids in MinorClosed Classes. Ann. Comb., 19(1):107-123, 2015. doi:10.1007/s00026-015-0251-3. 
17 Leslie Ann Goldberg and Mark Jerrum. A polynomial-time algorithm for estimating the partition function of the ferromagnetic ising model on a regular matroid. SIAM J. Comput., 42(3):1132-1157, 2013. doi:10.1137/110851213.

18 Alexander Golynski and Joseph Douglas Horton. A polynomial time algorithm to find the minimum cycle basis of a regular matroid. In Algorithm Theory - SWAT 2002, 8th Scandinavian Workshop on Algorithm Theory, Turku, Finland, July 3-5, 2002 Proceedings, volume 2368 of Lecture Notes in Computer Science, pages 200-209. Springer, 2002.

19 Jiong Guo, Rolf Niedermeier, and Sebastian Wernicke. Parameterized complexity of generalized vertex cover problems. In Algorithms and Data Structures, 9th International Workshop, WADS 2005, Waterloo, Canada, August 15-17, 2005, Proceedings, volume 3608 of Lecture Notes in Computer Science, pages 36-48, 2005. doi:10.1007/11534273_5.

20 Moritz Hardt and Ankur Moitra. Algorithms and hardness for robust subspace recovery. In Proceedings of the 26th Annual Conference on Learning Theory (COLT), volume 30 of JMLR Proceedings, pages 354-375. JMLR.org, 2013.

21 Petr Hlinený. Branch-width, parse trees, and monadic second-order logic for matroids. $J$. Comb. Theory, Ser. B, 96(3):325-351, 2006. doi:10.1016/j.jctb.2005.08.005.

22 Petr Hlinený and Sang-il Oum. Finding branch-decompositions and rank-decompositions. SIAM J. Comput., 38(3):1012-1032, 2008. doi:10.1137/070685920.

23 Jisu Jeong, Eun Jung Kim, and Sang-il Oum. Constructive algorithm for path-width of matroids. In Proceedings of the Twenty-Seventh Annual ACM-SIAM Symposium on Discrete Algorithms, SODA 2016, Arlington, VA, USA, January 10-12, 2016, pages 16951704. SIAM, 2016. doi:10.1137/1.9781611974331.ch116.

24 Gwenaël Joret and Adrian Vetta. Reducing the rank of a matroid. Discrete Mathematics $\&$ Theoretical Computer Science, 17(2):143-156, 2015. URL: http://www.dmtcs.org/ dmtcs-ojs/index.php/dmtcs/article/view/2334.

25 Ken-ichi Kawarabayashi and Mikkel Thorup. The minimum k-way cut of bounded size is fixed-parameter tractable. In FOCS 2011, pages 160-169. IEEE Computer Society, 2011.

26 Leonid G. Khachiyan, Endre Boros, Khaled M. Elbassioni, Vladimir Gurvich, and Kazuhisa Makino. On the complexity of some enumeration problems for matroids. SIAM J. Discrete Math., 19(4):966-984, 2005. doi:10.1137/S0895480103428338.

27 Daniel Král'. Decomposition width of matroids. Discrete Applied Mathematics, 160(6):913923, 2012. doi:10.1016/j.dam.2011.03.016.

28 Dániel Marx. Parameterized graph separation problems. Theor. Comput. Sci., 351(3):394406, 2006. doi:10.1016/j.tcs.2005.10.007.

29 Jesper Nederlof. Fast polynomial-space algorithms using inclusion-exclusion. Algorithmica, 65(4):868-884, 2013. doi:10.1007/s00453-012-9630-x.

30 Sang-il Oum and Paul D. Seymour. Approximating clique-width and branch-width. $J$. Comb. Theory, Ser. B, 96(4):514-528, 2006. doi:10.1016/j.jctb.2005.10.006.

31 Sang-il Oum and Paul D. Seymour. Testing branch-width. J. Comb. Theory, Ser. B, 97(3):385-393, 2007. doi:10.1016/j.jctb.2006.06.006.

32 James Oxley. Matroid theory, volume 21 of Oxford Graduate Texts in Mathematics. Oxford University Press, Oxford, second edition, 2011. doi : 10.1093/acprof : oso/9780198566946. 001.0001.

33 Marcin Pilipczuk, Michal Pilipczuk, Piotr Sankowski, and Erik Jan van Leeuwen. Network sparsification for steiner problems on planar and bounded-genus graphs. In 55th IEEE Annual Symposium on Foundations of Computer Science, FOCS 2014, Philadelphia, PA, USA, October 18-21, 2014, pages 276-285. IEEE Computer Society, 2014. doi:10.1109/ FOCS. 2014.37.

34 Paul D. Seymour. Decomposition of regular matroids. J. Comb. Theory, Ser. B, 28(3):305359, 1980. doi:10.1016/0095-8956(80)90075-1. 
35 Paul D. Seymour. Matroid minors. In Handbook of combinatorics, Vol. 1, 2, pages 527-550. Elsevier, Amsterdam, 1995.

36 Klaus Truemper. Max-flow min-cut matroids: Polynomial testing and polynomial algorithms for maximum flow and shortest routes. Math. Oper. Res., 12(1):72-96, 1987. doi:10.1287/moor.12.1.72.

37 Klaus Truemper. Matroid decomposition. Academic Press, 1992.

38 Alexander Vardy. The intractability of computing the minimum distance of a code. IEEE Trans. Information Theory, 43(6):1757-1766, 1997. doi:10.1109/18.641542.

39 D. J. A. Welsh. Combinatorial problems in matroid theory. In Combinatorial Mathematics and its Applications (Proc. Conf., Oxford, 1969), pages 291-306. Academic Press, London, 1971.

40 Mingyu Xiao and Hiroshi Nagamochi. An FPT algorithm for edge subset feedback edge set. Inf. Process. Lett., 112(1-2):5-9, 2012. doi:10.1016/j.ipl.2011.10.007. 